\title{
ABSOLUTE CONTINUITY AND WEAK COMPACTNESS
}

\author{
BY CONSTANTIN NICULESCU
}

Communicated by Robert Bartle, May 27, 1975

In this paper we discuss a class of $a$ priori inequalities of a type appearing frequently in linear partial differential equations and outline the connection with the class of all weakly compact operators. The point of departure is the wellknown result due to Bartle, Dunford and Schwartz [1] asserting the existence of a control measure for each weakly compact operator given on a space $C(S)$ and the reader can remark easily that our main result (Theorem 4) provides a more precise estimate than that obtained in [1]. Estimates of the same type hold also for $p$-absolutely summing operators $(1 \leqslant p<\infty)$ as well as for the compact ones, which shows an interesting connection between these classes. Finally we extend the well-known criterion of compactness in a space $l_{p} \quad(1 \leqslant p<\infty)$ by proving that, on a space which does not contain an isomorph of $l_{1}$, the compact operators are completely determined by certain $a$ priori inequalities.

The details will appear elsewhere.

Let $X, Y$ be two Banach spaces and let $p$ be a continuous seminorm on $X$. The following definition extends considerably a basic concept in the theory of vector measures:

1. Definition. An operator $T \in L(X, Y)$ is said to be absolutely continuous with respect to $p$ (i.e., $T \ll p$ ) if the following equivalent conditions hold:

$\left(\mathrm{AC}_{1}\right)$ for every $\epsilon>0$ there is a $\delta=\delta(\epsilon)>0$ such that if $\|x\| \leqslant 1, p(x)<$ $\delta$, then $\|T x\|<\epsilon$;

$\left(\mathrm{AC}_{2}\right)$ for every $\epsilon>0$ there is a $\delta=\delta(\epsilon)>0$ such that $\|T x\| \leqslant \epsilon\|x\|+$ $\delta p(x)$ whenever $x \in X$;

$\left(\mathrm{AC}_{3}\right)$ given a bounded sequence $\left\{x_{n}\right\}_{n} \subset X$, then either there exists a positive constant $c>0$ such that $\left\|T\left(x_{n}\right)\right\| \leqslant c p\left(x_{n}\right)$ for all $n \in \mathrm{N}$ or there exists a subsequence $\left\{x_{n_{k}}\right\}_{k}$ such that $T\left(x_{n_{k}}\right) \rightarrow 0$.

This notion is implicit in many papers on partial differential equations in which case $p$ is associated with an inner product. Inequalities such as Gårding's or Friedrichs' are consequences of the fact that suitable operators are absolutely continuous.

A local condition for absolute continuity was introduced in [5] for operators given on Banach lattices and used to describe the structure of Banach lattices

AMS (MOS) subject classifications (1970). Primary 46G10.

Key words and phrases. Absolute continuity, absolute summability, weak compactness. Copyright $\odot$ 1975, American Mathematical Society 
having an order continuous topology. (See also [6].)

In the following, by an absolutely continuous operator (abbreviated a.c. operator) we shall always mean an operator which is a.c. with respect to a prenuclear seminorm $p$ [i.e., a seminorm $p$ such that

$$
p(x) \leqslant \int\left|\left\langle x, x^{*}\right\rangle\right| d \mu\left(x^{*}\right),
$$

where $\mu$ is a positive Radon measure on the unit ball of $X^{*}$ ].

The results below yield that each $r$-absolutely summing operator $(1 \leqslant r<$ $\infty)$ and each compact operator is a.c. Using the Riesz convexity theorem one can obtain the same conclusion for inclusion mappings $i_{q}: l_{1} \rightarrow l_{q}, 1<q \leqslant \infty$ (communicated to the author by D. Lewis).

2. REMARK. An operator whose restriction to an infinite dimensional subspace is an isomorphism into cannot be a.c. (Use Dvoretzky's-Rogers' result in [2] and $\left(\mathrm{AC}_{2}\right)$.) In particular, if $i \in L(X, Y)$ is an isomorphism into and $T \in L(X, Y)$ is a.c., then $\operatorname{Ker}(i+T)$ is finite dimensional.

The product of an a.c. operator and a continuous mapping is always a.c., and thus the a.c. operators constitute a Banach ideal of operators in the sense of Pietsch. An a.c. operator maps bounded sequences into weak Cauchy sequences (use the main result in [7]) and Lebesgue's theorem on dominated convergence yields that each a.c. operator maps weak Cauchy sequences into convergent sequences. Therefore the product of two a.c. operators is a compact one.

3. REMARK. An operator $T$ is a.c. iff $T^{* *}$ is a.c. Combining with the results above we check that every a.c. operator on an $L_{\infty}$-space (i.e., a Banach space whose topological dual is isomorphic to a complemented subspace of some $L_{1}(\mu)$ ) is weakly compact. The converse is a consequence of the following:

4. Theorem. Let $X$ be an $L_{\infty}$-space and let $A$ be a bounded subset of $X^{*}$. The following assertions are equivalent:

(a) A is $\sigma\left(X^{*}, X^{* *}\right)$ relatively compact;

(b) there is a prenuclear seminorm $p$ on $X$ such that $A \ll p$ uniformly [i.e., for every $\epsilon>0$ we can find a $\delta=\delta(\epsilon)>0$ such that $\|x\| \leqslant 1, p(x)<\delta$ implies that $\left.\sup \left\{\left|\left\langle x, x^{*}\right\rangle\right|, x^{*} \in A\right\}<\delta\right]$.

5. COROLlARY. An operator given on an $\mathrm{L}_{\infty}$-space is weakly compact iff it is a.c.

6. CoRollary. Each compact operator is a.c.

Use Corollary 4 in $[4$, p. 24].

7. Corollary. Let $X$ be a Banach space which does not contain an isomorph of $l_{1}$. Then a bounded subset $A \subset X^{*}$ is relatively compact iff there exists a prenuclear seminorm $p$ on $X$ such that $A \ll p$ uniformly.

The assumption about $l_{1}$ is essential. In fact, if $X$ contains $l_{1}$ then there 
exists an integral operator $T \in L\left(X, L_{1}(0,1)\right)$ which is not compact.

\section{REFERENCES}

1. R. G. Bartle, N. Dunford and J. Schwartz, Weak compactness and vector measures, Canad. J. Math. 7 (1955), 289-305. MR 16, 1123.

2. A. Dvoretzky and C. A. Rogers, Absolute and unconditional convergence in normed linear spaces, Proc. Nat. Acad. Sci. U.S.A. 36 (1950), 192-197. MR 11, 525.

3. A. Grothendieck, Sur les applications linéaires faiblement compactes d'espaces du type C(K), Canad. J. Math. 5 (1953), 129-173. MR 15, 438.

4. - Résumé de la théorie métrique des produits tensoriels topologiques, Bol. Soc. Mat. São Paolo 8 (1953), 1-79 (1956). MR 20 \#1194.

5. C. Niculescu, Opérateurs absolument continus, Rev. Roumaine Math. Pures Appl. 19 (1974), 225-236.

6. - Lebesgue-Nikodym type theorems for operators given on Banach lattices, Rev. Roumaine Math. Pures Appl.

7. H. Rosenthal, $A$ characterization of Banach spaces containing l, Proc. Nat. Acad. Sci. U.S.A. 71 (1974), $2411-2413$.

DEPARTMENT OF MATHEMATICS, UNIVERSITY OF ILLINOIS, URBANA-CHAMPAIGN, URBANA, ILLINOIS 61801

Current address: Department of Mathematics, Institute of Mathematics, Academiei 14, Bucureşti, Romania 